\title{
Electron microscopes as educational tools: The use of a Scanning Electron Microscope to develop 3D models for educational programs.
}

\author{
Hurtado Padilla, Juan Pablo ${ }^{1 *}$ \\ ${ }^{1}$ Smithsonian National Museum of Natural History, Washington DC USA \\ * Corresponding author: hurtadopadillaj@si.edu
}

The Smithsonian's National Museum of Natural History (NMNH) has been working for decades in formal and informal scientific education. However, just recently, and thanks to the contribution of Thermo Fisher (previously FEI), the museum was able to launch a novel educational project: The use state-of-the-art optical and electron microscopes in conjunction with a Microscopy Educator, for the design and implementation of a variety of education programs aimed to diverse audiences.

One of these programs solved a problem we faced since the inception of the Department of Education at the Museum. How to use microscopic objects from the collection in educational programs? One of the pillars of the museum is the conservation of objects, thus the manipulation of these objects by the public was not an option. The solution was putting together science, technology and educational resources available in terms of using 3D reconstruction of images obtained with the scanning electron microscope, and its subsequent 3D printing, in this particular case one single scale from the nurse shark Ginglymostoma cirratum.

One single scale from Ginglymostoma cirratum was mounted in an aluminum stub and sputter coated with Pd/Au (Leica ACE600), this scale was then imaged in an ultra-high vacuum SEM (Thermo Fisher APREO). To obtain images that would be used in a 3D reconstruction, a protocol of image capture was created. The sample would be imaged at $0,10,20,30,40,50$ and 55 degrees of tilt for each 45 degrees of rotation obtaining 56 high-resolution images. These images where then processed in a specialized 3D reconstruction software (Agisoft's Metashape) to render a 3D model of the scale. A 3D model editing software was used to crop and clean the model and prepare it for printing (Autodesk's Meshmixer). The final steep for the 3D model involved the use of proprietary 3D printer software to test settings and printability (FormLabs' PreForm). Finally, the model was printed on a resin-based 3D printer (FormLabs' Form 2).

After printing, this 3D scale was used in the workshop "Shark Scales and Art," which highlighted the intersection between art, nature and science. The program allowed people to see, touch and feel a large size model of a microscopic object from the collection while learning about the marvelous advantages the shark scales morphology provides to these animals under the water [1], its historic uses [2] and how it can inspire us in science and art. This protocol is now being used regularly to offer higher quality educational programs in the Museum and there are plans to spread its application to other programs and institutions. 


\section{References:}

[1] Dean, B and Bhushan, B. Phil. TRans. R. Soc. A (2010) 638, 4775-4806

[2] Rick, T et al. Journal of Archeological Science (2002) Volume 29, Issue 2, 111-122

Acknowledgments:

We want to thank Thermo Fisher for providing the Electron microscope used for this project.
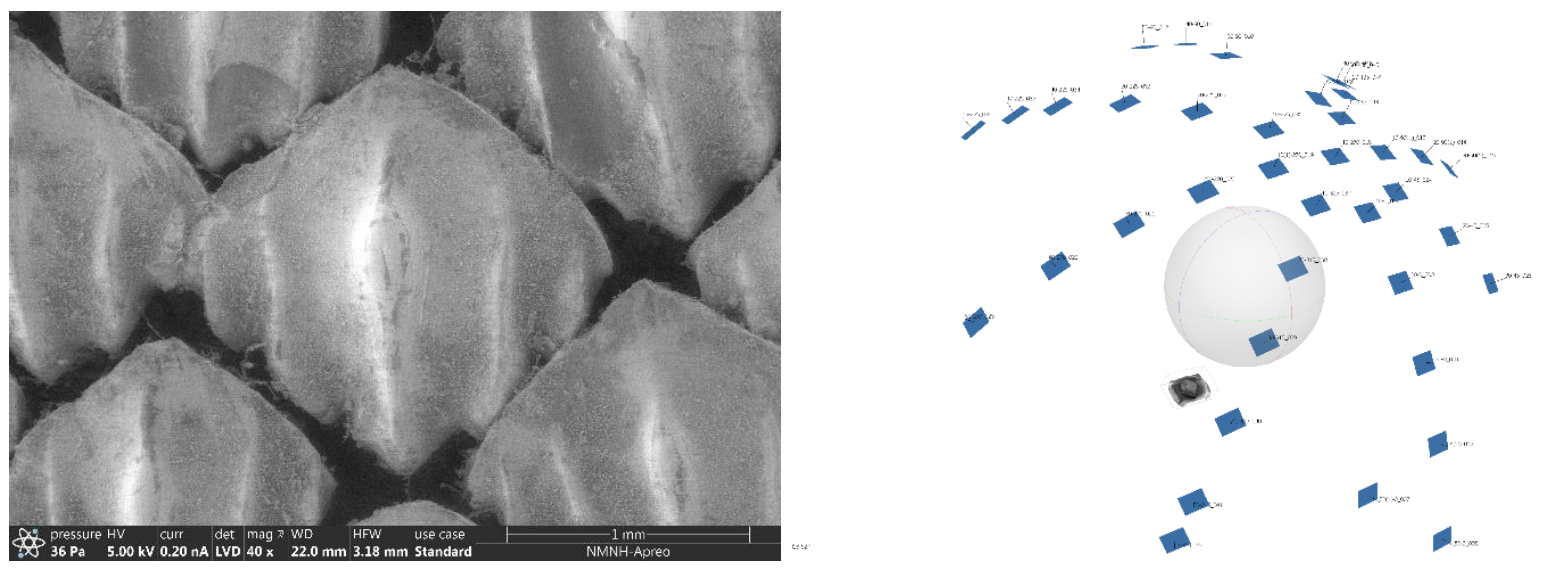

Figure 1. Image of the Nurse Shark skin (left). Relative position of images taken for 3D reconstruction (right)

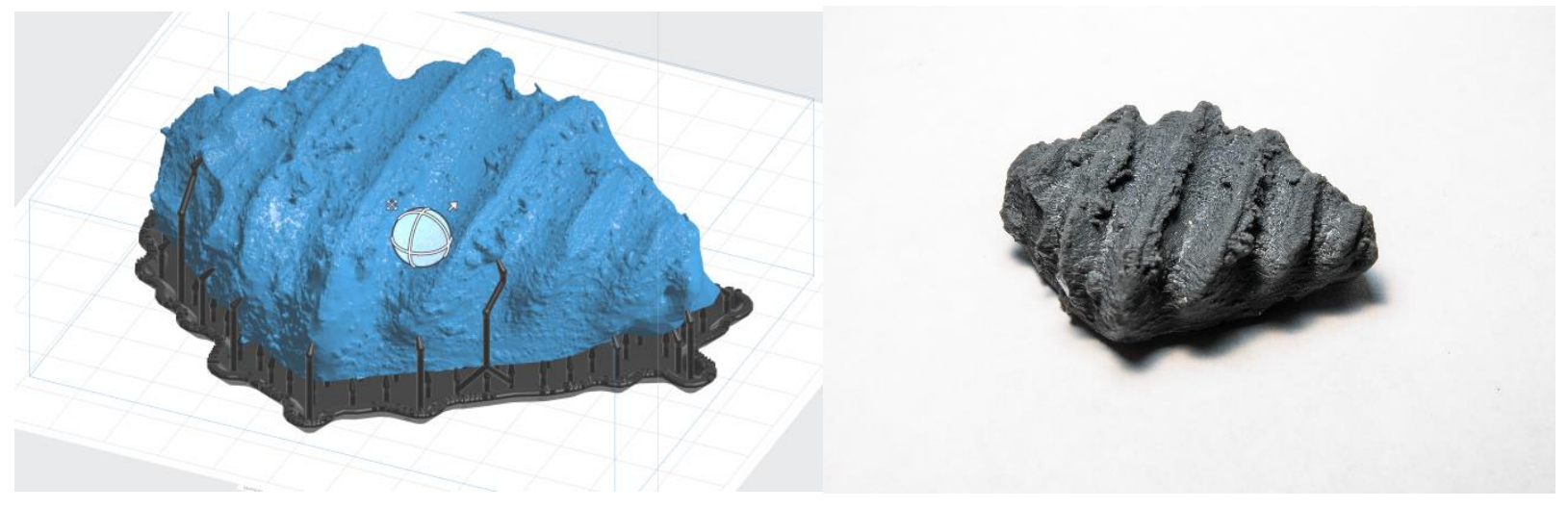

Figure 2. 3D model ready for 3D printing (left). Picture of 3D model printed (right) 